\title{
An Inventory Model with Dependent Product Demands AND \\ RETURNS
}

\author{
GUDRUN P. KIESMÜLLER AND ERWIN VAN DER LAAN
}

\begin{tabular}{|l|l|}
\hline \multicolumn{2}{|l|}{ ERIM REPORT SERIES RESEARCH IN MANAGEMENT } \\
\hline ERIM Report Series reference number & ERS-2001-16-LIS \\
\hline Publication & March 2001 \\
\hline Number of pages & 25 \\
\hline Email address corresponding author & elaan@fbk.eur.nl \\
\hline URL (electronic version) & http://www.eur.nl/WebDOC/doc/erim/erimrs20010327093400.pdf \\
\hline Address & Erasmus Research Institute of Management (ERIM) \\
& Rotterdam School of Management / Faculteit Bedrijfskunde \\
& Erasmus Universiteit Rotterdam \\
& P.O. Box 1738 \\
& 3000 DR Rotterdam, The Netherlands \\
& Phone: +31 10 408 1182 \\
& Fax: $\quad+31104089640$ \\
& Email: info@erim.eur.nl \\
& Internet: $\quad$ www.erim.eur.nl \\
\hline
\end{tabular}

Bibliographic data and classifications of all the ERIM reports are also available on the ERIM website:

www.erim.eur.nl 


\title{
ERASMUS RESEARCH INSTITUTE OF MANAGEMENT
}

\author{
REPORT SERIES \\ RESEARCH IN MANAGEMENT
}

\begin{tabular}{|c|c|c|}
\hline \multicolumn{3}{|c|}{ BIBLIOGRAPHIC DATA AND CLASSIFICATIONS } \\
\hline Abstract & \multicolumn{2}{|c|}{$\begin{array}{l}\text { In this paper an inventory model for a single reusable product is investigated, in which the } \\
\text { random returns depend explicitly on the demand stream. Further, the model distinguishes itsel } \\
\text { from most other research in this field by considering leadtimes and a finite planning horizon. We } \\
\text { show that neglecting the dependency between demands and returns of products may lead to bad } \\
\text { performance with respect to total average relevant costs. Additionally, our results enable us to } \\
\text { determine the minimal recovery probability or the minimal length of the planning horizon for which } \\
\text { reuse is profitable. }\end{array}$} \\
\hline \multirow{3}{*}{$\begin{array}{l}\text { Library of Congress } \\
\text { Classification } \\
\text { (LCC) }\end{array}$} & 5001-6182 & Business \\
\hline & 5201-5982 & Business Science \\
\hline & HD 40 & Inventory Policy \\
\hline \multirow{4}{*}{$\begin{array}{l}\text { Journal of Economic } \\
\text { Literature } \\
\text { (JEL) }\end{array}$} & M & Business Administration and Business Economics \\
\hline & M 11 & Production Management \\
\hline & R4 4 & Transportation Systems \\
\hline & M 11 & Production Management \\
\hline \multirow{4}{*}{$\begin{array}{l}\text { European Business Schools } \\
\text { Library Group } \\
\text { (EBSLG) }\end{array}$} & $85 \mathrm{~A}$ & Business General \\
\hline & $260 \mathrm{~K}$ & Logistics \\
\hline & $240 \mathrm{~B}$ & Information Systems Management \\
\hline & $280 \mathrm{~N}$ & Production planning \\
\hline \multicolumn{3}{|c|}{ Gemeenschappelijke Onderwerpsontsluiting (GOO) } \\
\hline \multirow[t]{4}{*}{ Classification GOO } & 85.00 & Bedrijfskunde, Organisatiekunde: algemeen \\
\hline & 85.34 & Logistiek management \\
\hline & 85.20 & Bestuurlijke informatie, informatieverzorging \\
\hline & 85.35 & Produktiemanagement \\
\hline \multirow[t]{3}{*}{ Keywords GOO } & \multicolumn{2}{|c|}{ Bedrijfskunde / Bedrijfseconomie } \\
\hline & \multicolumn{2}{|c|}{ Bedrijfsprocessen, logistiek, management informatiesystemen } \\
\hline & \multicolumn{2}{|c|}{ Voorraadbeheer, Stochastische modellen, Hergebruik } \\
\hline Free keywords & \multicolumn{2}{|c|}{ Reusable Products, Remanufacturing, Order-up-to Policy, Markov Chain } \\
\hline
\end{tabular}




\title{
An Inventory Model with Dependent Product Demands and Returns ${ }^{1}$
}

\author{
Gudrun P. Kiesmüller \\ Faculty of Technology Management \\ Eindhoven University of Technology \\ P.O. Box 513, Paviljoen F14 \\ NL-5600 MB Eindhoven \\ The Netherlands \\ Erwin A. van der Laan ${ }^{2}$ \\ Department of Decision and Information Sciences \\ Faculty of Business Administration \\ Erasmus University Rotterdam \\ P.O. Box 1738 \\ NL-3000 DR Rotterdam \\ The Netherlands
}

\begin{abstract}
In this paper an inventory model for a single reusable product is investigated, in which the random returns depend explicitly on the demand stream. Further, the model distinguishes itself from most other research in this field by considering leadtimes and a finite planning horizon. We show that neglecting the dependency between demands and returns of products may lead to bad performance with respect to total average relevant costs. Additionally, our results enable us to determine the minimal recovery probability or the minimal length of the planning horizon for which reuse is profitable.
\end{abstract}

Keywords: Reusable Products, Remanufacturing, Order-up-to Policy, Markov Chain

\footnotetext{
${ }^{1}$ The research presented in this paper makes up part of the research on re-use in the context of the TMR project REVersed LOGistics financially supported by the Europan Union (ERB 4061 PL 97-650) in which apart from Eindhoven University of Technology take part the Erasmus University Rotterdam $(\mathrm{NL})$, the Aristoteles University of Thessaloniki (GR), INSEAD (F), the Otto-von-Guericke-Universitaet Magdeburg (D) and the University of Piraeus (GR). The second author greatly acknowledges the financial support provided by the NWO.

${ }^{2}$ Corresponding Author: phone: +31-(0)10-4082763/2032, fax : +31-(0)10-4089010, e-mail: elaan@fac.fbk.eur.nl
} 


\section{Introduction}

A number of papers have studied inventory policies under consideration of random returns (see Fleischmann et al. (1997) for an overview), but most of these assume that the return process is independent of the demand process (see e.g. Inderfurth, 1997). This type of modeling may be good for situations in which no information about a dependence structure is available or situations with many different sources for the returns. But there are also situations in which a model with a dependence structure between the demands and the returns seems to fit better. We have such situations in the case of rented or leased products, or if delivered items are returned to the original manufacturer only. We can also think about packaging and transportation materials.

In this paper we develop and investigate an inventory model for a single, reusable product in which the random returns depend explicitly on the demand stream. A similar situation is also discussed in Cohen and Pierskalla (1980), but without considering backorders. Additionally, they do not consider the leadtime for purchasing. Also Yuan and Cheung (1998) consider a model with dependent returns, but without a purchasing leadtime. We relax this assumption and allow a positive purchasing leadtime.

Silver and Kelle (1989) determine an optimal purchasing policy for reusable containers by transferring the stochastic model in a deterministic one. We use a Markov-Chain approach in order to determine the optimal order-up-to policy with respect to total average relevant cost.

Although in many papers the long-term behaviour of the inventory is investigated (see e.g. Van der Laan and Salomon, 1997) we consider a finite planning horizon. The reason for this is that nowadays, the life-cycle of products is getting shorter and shorter, because of fast changing trends and new developments. This can be observed, in particular, for electronic products. Therefore, inventory models for short-term control have to be investigated and preferably objective functions should be used where no steady state assumptions are necessary.

In this paper we show that there is a great difference between a situation with independent demands and returns and a situation, where the returns depend on the demand. Therefore, neglecting the dependency of the returns on demands may lead to poor policy performance. We show how to compute the optimal order-up-to level for a situation with dependent returns. In such a situation the optimal inventory level is less than in the other case and the average relevant costs can be reduced by using the information about the dependence structure. The influence of the return probability and the length of the planning horizon on policy performance is investigated. Additionally, we compare a system with product returns with a system without product returns. Our results enable to answer questions regarding the profitability of product reuse in relation with return probability and time-in-market.

The paper is further organized as follows. In Section 2 we start with a detailed description of the model and its underlying assumptions. In Section 3 we develop an approximation to the total average costs, which we use as the objective function, followed by a numerical study (Section 4). Finally, we summarize our results and conclusions and give an outlook for further research. 


\section{Model Definition and Assumptions}

We consider a single, reusable product. Because of fast changing trends in the market, this product is planned to be produced only for a limited planning horizon $T$ which is the same as the time in market. Practical limitations are the reason for reviewing the inventory only periodically. We assume, that the length of one period is known and constant. Without loss of generality, we take the length of a period equal to one, and the periods are numbered by $t=1,2, \ldots, T$.

We assume that the demands per period $t, D_{t}$, are mutually independent and follow a Poisson distribution with parameter $\lambda_{t}$ :

$$
P\left(D_{t}=i\right)=e^{-\lambda_{t}} \frac{\left(\lambda_{t}\right)^{i}}{i !} \quad i \in N_{0}, t=1,2, \ldots, T-1, T
$$

All demands that cannot be fulfilled immediately are backordered. All demands that are outstanding at the end of the planning horizon are lost. Figure 1 gives an illustration of the reuse network.

\section{INSERT FIGURE 1}

We assume a constant probability, $p_{l}$, that an item is lost because a customer has not returned it to the manufacturer after use. Returned items are either remanufactured or disposed depending on their quality. With the probability $p_{d}$ a returned item cannot be remanufactured because of poor quality, and has to be disposed. With probability $1-p_{d}$ an item is remanufactured. Only at the end of the planning horizon all the returned items have to be disposed, because there is no longer demand for them. After remanufacturing, the item is as good as new and can be sold at the same market as newly manufactured products. Therefore, there is a common servicable inventory for both new and remanufactured items, since we do not need to distinguish between them.

All leadtimes are assumed to be constant: the time the item is in use by the customer $L_{1}$, the transportation time $L_{2}$, the remanufacturing time $L_{3}$ and the purchasing leadtime $L$. For computational reasons we assume

$$
L=L_{1}+L_{2}+L_{3}
$$

With the above assumptions we have all the necessary information to determine the probability distribution of the returns in one period. The returns in period $t$ are defined as the number of used and remanufactured items which enter the inventory in period $t$. Obviously, the returns in period $t$ are dependent on the demands $L$ periods before.

With respect to the planning horizon $T \geq 2 L$ we can distinguish three different time phases (Figure 2):

- Phase I: Start Phase $(1 \leq t \leq L)$

During this phase there are no remanufactured or new items delivered to the inventory. Therefore, the stock on hand is always decreasing in each of these periods. 
- Phase II: Main Phase $(L+1 \leq t \leq T-L)$

In this phase both remanufactured and new items enter the inventory. The stock on hand can decrease and increase during each period.

- Phase III: End Phase $(T-L+1 \leq t \leq T)$

In this phase no procurement orders take place. At the end of this phase all the items that are returned to the company are disposed, so that no used items enter the inventory.

\section{INSERT FIGURE 2}

Although it is not the optimal policy, we consider a periodic order-up-to policy, since it is widely used in practice and very effective. For recent results on optimal policies in the context of inventory systems with returns we refer to [3] and [4].

At the beginning of the planning horizon the inventory is filled up with $A$ items. An order is placed at the beginning of a period if the inventory position, which we will define later on, is smaller than $S$ in order to fill it up to this level $S$. This policy only depends on two parameters, i.e., the inventory level $S$ and the number of items $A$ that are in the inventory at the beginning. The set of all possible order-up-to policies is given by

$$
\mathcal{K}=\{(A, S) \mid A \in \mathbb{N}, S \in \mathbb{N}\}
$$

In order to compare different parameter sets $(A, S) \in \mathcal{K}$ for the review policy we use the average total relevant cost as an objective function. They are defined as the sum of the average relevant cost in each period $C_{t}$, the starting cost for filling up the inventory before the first period $C_{0}$, and the average cost at the end of the planning horizon for the disposal of the remaining items $C_{E}$ :

$$
C_{R}(A, S):=C_{0}(A)+\sum_{t=1}^{T} C_{t}(A, S)+C_{E}(A, S) \quad \forall(A, S) \in \mathcal{K}
$$

The optimal policy $\left(A_{\text {opt }}, S_{\text {opt }}\right) \in \mathcal{K}$ is defined as the parameter set that minimizes expression (4).

The starting costs only depend on the number of items in the inventory at the beginning of the planning horizon and on some fixed $\operatorname{costs} c_{A}$ :

$$
C_{0}(A):=C_{0}(A)=c_{A}+c_{P} \cdot A
$$

The average costs at the end of the planning horizon $C_{E}(A, S)$ are determined later on. The average relevant cost in one period are composed of the average costs for procurement, backorders and stockkeeping.

We assume that the costs are proportional to the number of items and that holding and backorder costs are charged at the end of each period. We get:

$$
C_{t}(A, S)=c_{P} E\left[O_{t}\right]+c_{B} E\left[X_{t}^{-}\right]+c_{H} E\left[X_{t}^{+}\right],
$$


where the random variables are defined as follows:

$O_{t}$ : Number of procured items at the beginning of period $t$

$X_{t}^{-} \quad$ : Number of backorders at the end of period $t$

$X_{t}^{+} \quad$ : Stock on hand at the end of period $t$

The cost parameters are defined as:

$c_{P} \quad:$ Procurement cost per item

$c_{H} \quad$ : Holding cost per item per period

$c_{B}:$ Backorder cost per item per period

For the description of the inventory process it is necessary to introduce some additional random variables:

$X_{t} \quad$ : Net stock at the end of period $t ;\left(X_{t}:=X_{t}^{+}-X_{t}^{-}\right)$

$I_{t} \quad$ : Inventory position at the beginning of period $t$ before a procurement order

$R_{t}$ : Number of remanufactured items that enter the servicable inventory in pe$\operatorname{riod} t$

Additionally, we define $p_{r}$ as the probability that an item is recovered, which means that it is returned to the manufacturer and it can be remanufactured:

$$
p_{r}:=\left(1-p_{l}\right)\left(1-p_{d}\right)
$$

\section{Determination of the Objective Function}

In order to determine the average relevant costs in one period we first introduce the inventory position $I_{t}$ and illustrate the differences between the dependent and the independent case. Then we are able to determine the optimization problem.

\subsection{The Inventory Position $I_{t}$}

In the model described in Section 2, a strong relation between the demands in period $t$ and the returns in period $t+L$ is given. If all demands are fulfilled in period $t$, then the distribution function of the random variable $R_{t+L}$ is only determined by the distribution function of the random variable $D_{t}$ and the recovery probability $p_{r}$. Otherwise it is also dependent on the number and the duration of backorders. Let $\hat{R}_{t}$ be a random variable, defined as the number of returns in period $t+L$ under the condition that all the demands in period $t$ are fulfilled. Then we can use $\hat{R}_{t}$ as an approximation for $R_{t+L}$ in situations with a high service level.

The strong relation between the demands in period $t$ and the returns in period $t+L$ is also important for practice. If the demands in period $t$ are known, we have some information about the returns $L$ periods later. This information should be used for the procurement decision in period $t$. In order to take this aspect into account we define the inventory position $I_{t}$, at the beginning of period $t$ before ordering, as the stock on hand minus backorders plus the outstanding orders plus the outstanding returns. Because of the high 
service level we get as an approximation for the inventory position for $1 \leq t \leq T-1$

$$
I_{t+1}:=X_{t}+\sum_{i=0}^{L-1} O_{t-i}+\sum_{i=0}^{L-1} \hat{R}_{t-i},
$$

or the following recursive equation:

$$
I_{t+1}=I_{t}+O_{t}-D_{t}+\hat{R}_{t}
$$

This means that the inventory position in period $t+1$ is given by the inventory position of the period before, the procurements and the number of demands in period $t$, and the number of fulfilled demands that will return to the inventory $L$ periods later. Therefore, $I_{t}$ describes a non-homogeneous Markov-Chain, because the inventory position in period $t+1$ depends only on the period before. The state space of this Markov-Chain is discrete and infinite.

If we consider the dependence of the demands and the returns, then $D_{t}-\hat{R}_{t}$ is always nonnegative, since obviously it is not possible to have more returns in period $t+L$ than there are demands in period $t$. It follows from (9) that the inventory position never exceeds $S$, so that we have $I_{t} \in\{i \mid i \leq S\}, 1 \leq t \leq T$. As a contrast, if the dependence of demands and returns is not considered, then $D_{t}-\hat{R}_{t}$ can also be negative, so that $I_{t} \in \mathbb{Z}$.

For a description of the system we need the state probabilities $\nu_{j}(t)$, which are the probabilities that the inventory position in period $t$ is equal to $j$ :

$$
\nu_{j}(t):=P\left(I_{t}=j\right) \quad 1 \leq t \leq T, j \in \mathbb{Z}
$$

At the beginning of period 1 , both the inventory position and the stock on hand have the value $A$, which leads to

$$
\nu_{A}(1)=1, \quad \text { and } \quad \nu_{j}(1)=0 \quad \forall j \neq A
$$

All the other state probabilities are given by the recursive formula

$$
\nu_{j}(t+1)=\sum_{k=-\infty}^{\infty} p_{k, j}(t) \nu_{k}(t)
$$

where $p_{k, j}(t)$ denotes the transition probability to go from state $k$ in period $t$ to state $j$ in period $t+1$, i.e.,

$$
p_{k, j}(t):=P\left(I_{t+1}=j \mid I_{t}=k\right) \quad 1 \leq t \leq T-1, j, k \in \mathbb{Z} .
$$

For the transition probabilities we get different formulas for the dependent and the independent case (see Appendix 1.3 and 2.3). They are mainly determined by the distribution of the random variables $D_{t}-\hat{R}_{t}$. In case of dependent returns this difference is Poisson distributed with parameter $\lambda_{t}\left(1-p_{r}\right)$ while in the other case with independent returns another distribution is derived (see Appendix 1.1 and 2.1). 


\subsubsection{Numerical Illustration}

In Figure 3 and 4 we illustrate the different behaviour for one sample path of the inventory position in the case of dependent returns and independent returns as a function of the time $t$ for different values of the recovery probability $p_{r}$.

\section{INSERT FIGURE 3}

In Figure 3, the dependent case, it can be seen, that the inventory position never exceeds the level $S$. With decreasing $p_{r}$ the variance of the inventory position is increasing. For the independent case the inventory position is given in Figure 4 for the same parameter values.

\section{INSERT FIGURE 4}

Both figures show that the behaviour of the inventory position is quite different in the dependent and the independent case. Because of the independence there can be more returns in one period than demands. This leads to an inventory position larger than $S$. Not surprisingly, this happens more often if the recovery probability is large. Additionally, the variance of $D_{t}-\hat{R}_{t}$ is much larger in the independent case than in the dependent case (see Table 1), which has a large influence on the variance of the inventory position.

\section{INSERT Table 1}

\subsection{The Objective Function}

In order to get a formula for the objective function we have to determine the average shortage and holding costs in period $t$ which are given for all $t=1,2, \ldots, T-1, T$ by

$$
c_{H} E\left[X_{t}^{+}\right]+c_{B} E\left[X_{t}^{-}\right]=c_{H} \sum_{i=1}^{\infty} i \cdot P\left(X_{t}=i\right)+c_{B} \sum_{i=1}^{\infty} i \cdot P\left(X_{t}=-i\right)
$$

where the probability distribution of the net stock $P\left(X_{t}=i\right)$ is given in the Appendix (1.4 and 2.4). Again, this distribution is different in case of dependent and independent returns.

Additionally, we have to consider the average procurement cost in the objective function. In the case of an order-up-to policy different procurement sizes are possible which leads to an average procurement size:

$$
E\left[O_{t}\right]= \begin{cases}0 & t=1 \\ \sum_{k=-\infty}^{S-1}(S-k) \nu_{k}(t) & 2 \leq t \leq T-L \\ 0 & T-L+1 \leq t \leq T\end{cases}
$$


The formula for the average number of orders is equal for the dependent and the independent scenario, but the numerical values for the state probabilities $\nu_{k}(t)$ will be different.

With (14) and (15) the average costs in one period $C_{t}(A, S)$ can be computed for $t=$ $1,2, \ldots, T$.

At the end of the planning horizon the company is responsible for the items that are still in the reuse network. They have to be transported back and must be disposed of. They also have to dispose of the items that are remaining in the inventory. We have

$$
C_{E}(A, S)=c_{D}\left(E\left[X_{T}^{+}\right]+\left(1-p_{l}\right) \sum_{i=0}^{L_{1}+L_{2}-1} \lambda_{T-i}\right)+c_{T} \cdot\left(1-p_{l}\right) \sum_{i=1}^{L_{1}-1} \lambda_{T-i} .
$$

Now that we have determined all the necessary expectations, we can calculate the objective function $C_{R}(A, S)$ for arbitrary parameter sets $(A, S) \in \mathcal{K}$. In case of a high service level a nearly optimal policy can be found solving the following problem:

$$
\min _{(A, S) \in \mathcal{K}} C_{R}(A, S)
$$

Because of the difficult structure of the objective function, the optimization problem is only solved numerically.

\section{Numerical Study}

Before we discuss the influence of the recovery probability and the length of the planning horizon on the economic profitability of product reuse (Section 4.2), we first compare the case of dependent returns with the case of independent returns. In our numerical examples we limit ourselves on constant demand and return rates since the focus of our investigations are effects resulting from the dependency and not from dynamic demand and return patterns. The following base case scenario for the system parameters is investigated. In the numerical studies we will vary some elements of this scenario.

$$
\begin{array}{lllll}
\lambda=10 & L_{1}=1 & c_{H}=1 \\
T=24 & L_{2}=1 & c_{B}=50 \\
p_{l}=0.2 & L_{3}=1 & c_{P}=40 \\
p_{d}=0.25 & L=3 & c_{A}=0
\end{array}
$$

Since we have assumed relatively high backorder cost, we get a high service level and our approach can be used.

\subsection{A Comparison Between Dependent Returns and Indepen- dent Returns}

In Table 2 and 3 the optimal policies and the average minimal relevant costs are given for different values of $p_{r}$ and $p_{l}$ in case of dependent returns and independent returns. 
Additionally the relative difference between both is given, which is defined as the difference between the average minimal costs in the independent and the dependent case divided by the average minimal costs in the dependent case.

\section{INSERT TABLE 2}

\section{INSERT TABLE 3}

It can be seen that the minimal average relevant costs for the independent case are larger than for the dependent case, and the difference between both is increasing with increasing recovery probability $p_{r}$. The difference is smaller, if the loss probability $p_{l}$ is larger.

Obviously, the optimal inventory level $S_{\text {opt }}$ and the optimal value $A_{\text {opt }}$ are increasing with decreasing recovery probability. The parameters of the optimal policy in the independent case are never smaller than in the dependent case. The reason for this is that the service level $\epsilon$ cannot be fulfilled with lower values of the decision parameters, because the variance of the returns is larger in the independent case. The influence of this effect is decreasing if the recovery probability is decreasing. Therefore, for small values of $p_{r}$ the difference between the dependent and the independent case is not significant, both for the optimal policy and for the minimal average relevant costs. At the same time the difference is substantial for large values of $p_{r}$. This is further illustrated in Figure 5.

\section{INSERT FIGURE 5}

In Table 4 the optimal policy and the minimum of the objective function (4) for the dependent and the independent scenario are shown for different values of the planning horizon $T$. The recovery probability is chosen as $p_{r}=0.95$.

\section{INSERT TABLE 4}

Again we can see that the minimal average relevant costs in the independent scenario are larger than in the dependent scenario. This holds also for the optimal policy parameters, which are nearly constant in $T$. Furthermore it can be seen that the relative difference of the minimal average relevant costs is non-decreasing in $T$.

\subsection{Economic Profitability of Reuse}

Here we compare, for the case of dependent returns, a system in which products can be reused with a system in which no products are reused. For the latter system we distinguish between two different situations:

$\mathcal{S}_{1}$ : The company is not responsible for the disposal of the items. This can be modeled by $p_{l}=1$. 
$\mathcal{S}_{2}$ : The company is responsible for a proper disposal of all returned items. This can be modeled by $p_{d}=1, p_{l}<1$.

For the comparison of the situations we have to include the average transportation, disposal and remanufacturing cost. Therefore, we have assumed the following cost parameter values

$$
\begin{aligned}
& c_{T}=1 \\
& c_{D}=5 \\
& c_{R}=2
\end{aligned}
$$

In Figure 6 we show the minimal average costs as a function of the recovery probability $p_{r}$ for different values of $p_{l}$. It can be seen that the minimal average costs decrease remarkably with increasing $p_{r}$. Moreover, we are able to compute the minimal recovery probability that is necessary to make reuse profitable in comparison with traditional manufacturing.

\section{INSERT FIGURE 6}

Next we investigate the influence of the length of the planning horizon on the economic profitability of product reuse. In order to do so, we compare the minimal average costs for the base scenario and those for a system without product reuse as a function of the planning horizon $T$ (Figure 7 ).

\section{INSERT FIGURE 7}

It can be seen that the recovery probability has a large influence on the economic profitability. If $p_{r}=0.6$ then a planning horizon of $T=50$ is not large enough to make reuse profitable in comparison with the situation in which the company is not responsible for disposal $\left(\mathcal{S}_{1}\right)$. Only if the company has to pay for the disposal $\left(\mathcal{S}_{2}\right)$, then reuse is profitable if the planning horizon is larger then 15 periods.

The situation is quite different if the recovery probability is larger $\left(p_{r}=0.75\right)$. In this case reuse is profitable after 17 periods (if the company is not responsible for disposal), or after 10 periods (if the company is responsible for the disposal).

\subsection{Practical Application}

In order to calculate the inventory position in period $t+1$ we always need the number of returns $L$ periods later (see equation (9)). So our calculations only hold for the rather idealistic situation in which all the necessary information is known at each time point. In practice no realization of the random variable $\hat{R}_{t}$ will be available in period $t$. For that reason an estimate of the number of returns has to be used. We suggest to use the expectation of the number of returns at time $t+L$ conditioned on the number of realized demands at time $t$, as an estimator for the returns:

$$
E\left[\hat{R}_{t} \mid D_{t}=n\right]=n p_{r}
$$


Then instead of using (9), the inventory position is computed recursively via

$$
I_{t+1}=X_{t}+\sum_{i=0}^{L-1} O_{t-i}+p_{r} \sum_{i=0}^{L-1} D_{t-i}
$$

and

$$
X_{t}=X_{t-1}+O_{t-L}-D_{t}+R_{t} ; \quad X_{0}=A
$$

Then realizations are available for all the necessary random variables for the computation of the inventory position. The equation for the inventory position is continuously adapted by replacing the forecasted values of the returns by the actual ones in the equation (20) for the net stock.

By means of simulation we show that our analytical results are very close to the results that we get if we implement the above procedure, which makes use of an estimation for the returns. In order to see the influence of the estimation we also have simulated a situation, in which the number of returns in period $t+L$ is exactly known in period $t$. For each set of (policy) parameters we have conducted 50,000 simulation runs. The results are shown in Table 5 and Table 6.

\section{INSERT TABLE 5}

\section{INSERT TABLE 6}

It shows that the simulation results from the situation in which the future returns are known and from the situation in which the returns are estimated, are very close. This holds for both the minimal average costs and the optimal policy $\left(A_{\text {opt }}, S_{\text {opt }}\right)$. Additionally, there are only small differences between our analytical results and the simulations. So, we can conclude that the procedure described by (19) and (20) leads to a near optimal policy. Of course the quality of the procedure depends on the estimator. If the variance of the estimator is larger, then the difference between the minimal average costs will also be larger. That is the reason why we can expect worse results for the independent case.

\section{Summary, Conclusions and Outlook}

In this paper we have developed a periodic review inventory system with product returns that depend explicitly on the demand stream. The system, which includes leadtimes and a finite planning horizon, is controlled by a fill-up policy. We showed how both the optimal policy and the minimal average total relevant cost depend on the recovery probability. Also we indicated the influence of the planning horizon on the minimal total average costs. The influence of the planning horizon on the optimal policy was found to be not significant. Central result of the paper however is that it pays off to use information about the dependence between the demand and return process. Not only minimal costs are generally lower, also the variance of inventory processes are considerably reduced. The potential benefits are higher with a higher recovery rate. 
Furthermore, our results enable us to determine the minimal recovery probability for an item that is necessary to make reuse beneficial. We can also determine the minimal planning horizon, for which a reusable product is profitable. Additionally, quantitative statements are possible about different products. Therefore, our results help to decide whether to reuse or not.

Although our model provides some insights, it depends on several limiting assumptions. It would be interesting to extend the model with a more general (stochastic) leadtime structure or more sophisticated control policies, which we get in case of set-up costs and if batch sizing is assumed. Additionally, non-stationary policies should be investigated. We have concentrated on Poisson distribution. Further research should be done in order to investigate other demand processes.

In our model we have only one inventory for the servicable items. An interesting extension would be a second inventory ahead of the remanufacturing process for the returned items. Another generalization that is worth to be discussed, is that a remanufactured item is not like a new one. In this case the recovery probability for an item is not constant but decreasing.

\section{References}

[1] M.A. Cohen and W.P. Pierskalla, 1980, A Dynamic Inventory System with Recycling, Naval Research Logistics Quarterly 27, 289 - 296.

[2] M. Fleischmann and J.M. Bloemhof-Ruwaard and R. Dekker and E. van DER LAAn and J.A.E.E. van NuEnen and L.N. van Wassenhove, 1997, Quantitative models for reverse logistics: A review, European Journal of Operational Research 103, 1-17.

[3] M. Fleischmann and R. KuiK, 1998, On optimal inventory control with stochastic item returns. Management Report Erasmus University Rotterdam 21.

[4] K. Inderfurth, 1997, Simple optimal replenishment and disposal policies for a product recovery system with leadtimes, OR Spektrum 19, 111-122.

[5] X. Yuan and K. Cheung, 1998, Modeling returns of merchandise in an inventory system, OR Spektrum 20, 147-154.

[6] E. VAn Der LAAN and M. SAlomon, 1997, Production planning and inventory control with remanufacturing and disposal, European Journal of Operational Research 102, 264-278.

[7] E.A. Silver and P. Kelle, 1989, Purchasing Policy of New Containers Considering the Random Returns of Previously Issued Containers, IIE Transactions 21/4, 340 354. 


\section{Appendix}

\section{Dependent Returns}

\subsection{The Distribution of the Returns $\hat{\mathbf{R}}_{t}$ and of $D_{t}-\hat{\mathbf{R}}$}

If $p_{r}$ denotes the probability that an item is recovered $\left(p_{r}=\left(1-p_{l}\right)\left(1-p_{d}\right)\right)$, then we get the following formula for the distribution of the returns, $\hat{R}_{t}$, for all $t=L+1, L+2, \ldots, T-1$ :

$$
\begin{aligned}
P\left(\hat{R}_{t}=i\right) & =\sum_{j=i}^{\infty} P\left(\hat{R}_{t}=i \mid D_{t-L}=j\right) P\left(D_{t-L}=j\right) \\
& =\sum_{j=i}^{\infty}\left(\begin{array}{l}
j \\
i
\end{array}\right) p_{r}^{i}\left(1-p_{r}\right)^{j-i} e^{-\lambda_{t-L}} \frac{\lambda_{t-L}^{j}}{j !} \\
& =e^{-\lambda_{t-L}} \frac{p_{r}^{i}}{i !} \sum_{j=i}^{\infty} \frac{j !}{(j-i) !}\left(1-p_{r}\right)^{j-i} \frac{\lambda_{t-L}^{j}}{j !} \\
& =e^{-\lambda_{t-L}} \frac{\left(p_{r} \lambda_{t-L}\right)^{i}}{i !} \sum_{j=i}^{\infty} \frac{\left(\lambda_{t-L}\left(1-p_{r}\right)\right)^{j-i}}{(j-i) !} \\
& =e^{-\lambda_{t-L}} \frac{\left(p_{r} \lambda_{t-L}\right)^{i}}{i !} e^{\left(1-p_{r}\right) \lambda_{t-L}} \\
& =e^{-p_{r} \lambda_{t-L}} \frac{\left(p_{r} \lambda_{t-L}\right)^{i}}{i !}
\end{aligned}
$$

Therefore, the number of returns in period $t$ is Poisson distributed with parameter $p_{r} \lambda_{t-L}$.

$$
\hat{R}_{t} \sim \operatorname{Po}\left(p_{r} \lambda_{t-L}\right) \quad t=L+1, L+2, \ldots, T-1
$$

The distribution is independent of $t$, if $\lambda_{t}=\lambda$. For $t=1,2, \ldots, L-1$ and for $t=T$ there are no returns, which means $\hat{R}_{t}=0$.

The distribution of the number of items that are not returned, $D_{t}-\hat{R}_{t}$, can be determined similarly. We get

$$
D_{t}-\hat{R}_{t} \sim \operatorname{Po}\left(\lambda_{t}\left(1-p_{r}\right)\right)
$$

1.2 The Distribution of $\sum_{i=0}^{L} D_{t-i}-\hat{R}_{t}$

In order to determine the distribution of $\sum_{i=0}^{L} D_{t-i}-\hat{R}_{t}$ we have to consider, that the random variables $\hat{R}_{t}$ and $D_{t-L}$ are dependent, while the other random variables not. We 
can write:

$$
\sum_{i=0}^{L} D_{t-i}-\hat{R}_{t}=\underbrace{D_{t-L}-\hat{R}_{t}}_{\text {dependent }}+\underbrace{\sum_{i=0}^{L-1} D_{t-i}}_{\text {independent }}
$$

$D_{t-L}-\hat{R}_{t}$ is Poisson distributed with parameter $\lambda_{t-L}\left(1-p_{r}\right)$

$$
D_{t-L}-\hat{R}_{t} \sim \operatorname{Po}\left(\lambda_{t}\left(1-p_{r}\right)\right)
$$

and $\sum_{i=0}^{L-1} D_{t-i}$ is Poisson distributed with parameter $\sum_{i=0}^{L-1} \lambda_{t-i}$

$$
\sum_{i=0}^{L-1} D_{t-i} \sim \operatorname{Po}\left(\sum_{i=0}^{L-1} \lambda_{t-i}\right)
$$

$D_{t-L}-\hat{R}_{t}$ and $\sum_{i=0}^{L-1} D_{t-i}$ itself are independent. Therefore the sum of these terms is again Poisson distributed, which means

$$
\sum_{i=0}^{L} D_{t-i}-\hat{R}_{t} \sim \operatorname{Po}\left(\lambda_{t-L}\left(1-p_{r}\right)+\sum_{i=0}^{L-1} \lambda_{t-i}\right)
$$

\subsection{The Transition Probabilities}

Because of the non-homogeneous Markov-Chain the transition probabilities are dependent on time.

- $\mathrm{t}=1$

The initial distribution (11) and the fact that $D_{t}-\hat{R}_{t}$ is always non-negative leads to

$$
p_{k, j}(1)= \begin{cases}P\left(D_{1}-\hat{R}_{1}=A-j\right), & j \leq A, k=A \\ 0, & \text { elsewhere }\end{cases}
$$

and the state probability

$$
\nu_{j}(2)=P\left(D_{1}-\hat{R}_{1}=A-j\right) .
$$

- $2 \leq \mathrm{t}<\mathrm{T}-\mathrm{L}$

In these periods from all the demands a portion will be returned to the inventory $L$ periods later, which means $\hat{R}_{t} \geq 0$. Additionally, the inventory position after an order is always $S$, so that $I_{t}+O_{t}=S$. Therefore, we have

$$
p_{k, j}(t)=P\left(D_{t}-\hat{R}_{t}=S-j\right) .
$$


These transition probabilities are independent of $k$, so that the state probabilities are given by:

$$
\nu_{j}(t+1)=P\left(D_{t}-\hat{R}_{t}=S-j\right)
$$

- $\mathrm{t}=\mathrm{T}-\mathrm{L}$

For $t=T-L$ we still have a procurement, but none of the items that are delivered to the customer will be returned to the serviceable inventory $\left(\hat{R}_{T-L}=0\right)$. Therefore, we get

$$
p_{k, j}(T-L)=P\left(D_{T-L}=S-j\right)
$$

and

$$
\nu_{j}(T-L+1)=P\left(D_{T-L}=S-j\right) .
$$

- $\mathrm{T}-\mathrm{L}+\mathbf{1} \leq \mathrm{t} \leq \mathrm{T}-\mathbf{1}$

In these periods we have again $\hat{R}_{t}=0$ but there are no orders $\left(O_{t}=0\right)$, which leads to

$$
p_{k, j}(t)=P\left(D_{t}=k-j\right) .
$$

\subsection{The Distribution of the Netstock}

Different formulas are needed for different periods.

- $1 \leq \mathrm{t} \leq \mathrm{L}$

Because of no returns in phase I, the net stock only depends on the demands and is monotonously decreasing. There are also no deliveries of new items. For $i \in \mathbb{Z}$ it holds that

$$
P\left(X_{t}=i\right)=P\left(\sum_{k=1}^{t} D_{k}=A-i\right), \quad t=1,2, \ldots, L .
$$

These probabilities can easily be computed, since the sum of independent Poisson distributed random variables is again Poisson distributed:

$$
\sum_{k=1}^{t} D_{k} \sim \operatorname{Po}\left(\sum_{k=1}^{t} \lambda_{k}\right)
$$

- $\mathrm{L}+\mathbf{1} \leq \mathrm{t} \leq \mathrm{T}-\mathbf{1}$

In phase II and III the net stock at the end of period $t$ is dependent on the inventory position in period $t-L$ :

$$
X_{t}=I_{t-L}+O_{t-L}+\hat{R}_{t}-\sum_{k=0}^{L} D_{t-k} \quad L+1 \leq t \leq T
$$

For the probabilities we have to distinguish between $t=L+1$ and $t \neq L+1$. 
- $\mathbf{t}=\mathbf{L}+1$

In this case there are no procurements $\left(O_{1}=0\right)$ and the inventory position in period 1 is given by $I_{1}=A$. Therefore, we get

$$
P\left(X_{L+1}=i\right)=P\left(\sum_{k=0}^{L} D_{L+1-k}-\hat{R}_{L+1}=A-i\right)
$$

- $\mathbf{t} \neq \mathbf{L}+\mathbf{1}$

In this case the probabilities for the net stock can be computed as follows:

$$
P\left(X_{t}=i\right)=P\left(\sum_{k=0}^{L} D_{t-k}-\hat{R}_{t}=S-i\right)
$$

- $\mathrm{t}=\mathrm{T}$

For $t=T$ the random variable $\hat{R}_{t}$ in (37) is equal to 0 and we get

$$
P\left(X_{T}=i\right)=P\left(\sum_{k=0}^{L} D_{T-k}=S-i\right)
$$

\section{Independent Returns}

In case of independent returns the distribution of the returns, $\hat{R}_{t}$, cannot be computed by means of the model and the assumptions. Therefore we have to assume a probability distribution for the returns. We suppose a Poisson distribution with parameter $p_{r} \lambda_{t-L}$ for the returns $\hat{R}_{t}$, which is similar to the dependent case, so that a comparison is possible.

\subsection{The Distribution of $D_{t}-\hat{R}_{t}$}

We get the following formula for the distribution of the items that are lost, $D_{t}-\hat{R}_{t}$, for all $t=1,2, \ldots, T-L-1$ :

- $i \geq 0$

$$
\begin{aligned}
P\left(D_{t}-\hat{R}_{t}=i\right) & =\sum_{j=0}^{\infty} P\left(D_{t}=j+i \mid \hat{R}_{t}=j\right) P\left(\hat{R}_{t}=j\right) \\
& =\sum_{j=0}^{\infty} e^{-\lambda_{t}} \frac{\lambda_{t}^{j+i}}{(j+i) !} e^{-p_{r} \lambda_{t}} \frac{\left(p_{r} \lambda_{t}\right)^{j}}{(j) !} \\
& =e^{-\lambda_{t}\left(1+p_{r}\right)} \sum_{j=0}^{\infty} \frac{\lambda_{t}^{j+i}}{(j+i) !} \frac{\left(p_{r} \lambda_{t}\right)^{j}}{(j) !}
\end{aligned}
$$


- $i<0$

$$
\begin{aligned}
P\left(D_{t}-\hat{R}_{t}=i\right) & =\sum_{j=-i}^{\infty} P\left(D_{t}=j+i \mid \hat{R}_{t}=j\right) P\left(\hat{R}_{t}=j\right) \\
& =\sum_{j=-i}^{\infty} e^{-\lambda_{t}} \frac{\lambda_{t}^{j+i}}{(j+i) !} e^{-p_{r} \lambda_{t}} \frac{\left(p_{r} \lambda_{t}\right)^{j}}{(j) !} \\
& =e^{-\lambda_{t}\left(1+p_{r}\right)} \sum_{j=-i}^{\infty} \frac{\lambda_{t}^{j+i}}{(j+i) !} \frac{\left(p_{r} \lambda_{t}\right)^{j}}{(j) !}
\end{aligned}
$$

2.2 The Distribution of $\sum_{\mathrm{i}=0}^{\mathrm{L}} \mathrm{D}_{\mathrm{t}-\mathrm{i}}-\hat{\mathbf{R}}_{\mathrm{t}}$

Now all the random variables in $\sum_{i=0}^{L} D_{t-i}-\hat{R}_{t}$ are independent. This leads to

- $i \geq 0$

$$
\begin{aligned}
P\left(\sum_{k=0}^{L} D_{t-k}-\hat{R}_{t}=i\right) & =\sum_{j=0}^{\infty} P\left(\sum_{k=0}^{L} D_{t-k}=j+i \mid \hat{R}_{t}=j\right) P\left(\hat{R}_{t}=j\right) \\
& =e^{-\left(p_{r} \lambda_{t}+\sum_{k=0}^{L} \lambda_{t-k}\right)} \sum_{j=0}^{\infty} \frac{\left(\sum_{k=0}^{L} \lambda_{t-k}\right)^{j+i}}{(j+i) !} \frac{\left(p_{r} \lambda_{t}\right)^{j}}{(j) !}
\end{aligned}
$$

- $i<0$

$$
\begin{aligned}
P\left(\sum_{i=0}^{L} D_{t-i}-\hat{R}_{t}=i\right) & =\sum_{j=-i}^{\infty} P\left(\sum_{i=0}^{L} D_{t-i}=j+i \mid \hat{R}_{t}=j\right) P\left(\hat{R}_{t}=j\right) \\
& =e^{-\left(p_{r} \lambda_{t}+\sum_{k=0}^{L} \lambda_{t-k}\right)} \sum_{j=-i}^{\infty} \frac{\left(\sum_{k=0}^{L} \lambda_{t-k}\right)^{j+i}}{(j+i) !} \frac{\left(p_{r} \lambda_{t}\right)^{j}}{(j) !}
\end{aligned}
$$

\subsection{The Transition Probabilities}

Also in the case of independent returns the transition probabilities are dependent on time, but additionally they are dependent on the inventory position.

- $\mathrm{t}=1$

$$
p_{k, j}(1)= \begin{cases}P\left(D_{1}-\hat{R}_{1}=A-j\right) & k=A \\ 0 & k \neq A\end{cases}
$$


and

$$
\nu_{j}(2)=P\left(D_{1}-\hat{R}_{1}=A-j\right)
$$

In contrast with the dependent case, $j$ can be larger than $S$, which means that the inventory position can also increase.

- $2 \leq \mathrm{t}<\mathrm{T}-\mathrm{L}$

In these periods we have $\hat{R}_{t} \geq 0$. Depending on the inventory position we get

$$
p_{k, j}(t)= \begin{cases}P\left(D_{t}-\hat{R}_{t}=S-j\right) & k \leq S \\ P\left(D_{t}-\hat{R}_{t}=k-j\right) & k>S\end{cases}
$$

These transition probabilities are only independent of $k$, if the inventory position is less than $S$, which means that an order takes place.

- $\mathrm{t}=\mathrm{T}-\mathrm{L}$

For $t=T-L$ we still may have a procurement, but $\hat{R}_{T-L}=0$. Therefore we get

$$
p_{k, j}(T-L)= \begin{cases}P\left(D_{T-L}=S-j\right) & k \leq S \\ P\left(D_{T-L}=k-j\right) & k>S\end{cases}
$$

- $\mathrm{T}-\mathrm{L}+\mathbf{1} \leq \mathrm{t} \leq \mathrm{T}-\mathbf{1}$

Here we have again $\hat{R}_{t}=0$, but additionally we have $O_{t}=0$, which leads to

$$
p_{k, j}(t)=P\left(D_{t}=k-j\right) .
$$

\subsection{The Probability Distribution of the Netstock}

- $1 \leq \mathrm{t} \leq \mathrm{L}$

For the first periods we get the same probabilities as in the dependent case (equation 27).

- $\mathrm{L}+\mathbf{1} \leq \mathrm{t} \leq \mathrm{T}-\mathbf{1}$

For these periods we have the same equation for the net stock as in the dependent case (equation 37), but we get different probabilities. We have to distinguish between $t=L+1$ and $t \neq L+1$.

- $\mathrm{t}=\mathrm{L}+\mathbf{1}$

$$
P\left(X_{L+1}=i\right)=P\left(\sum_{k=0}^{L} D_{L+1-k}-\hat{R}_{L+1}=A-i\right)
$$


- $\mathbf{t} \neq \mathbf{L}+\mathbf{1}$

In this case the probabilities for the net stock are dependent on the procurement size and on the inventory position. They can be computed as follows:

$$
\begin{aligned}
P\left(X_{t}=i\right)= & \sum_{k=S}^{\infty} P\left(\sum_{j=0}^{L} D_{t-j}-\hat{R}_{t}=k-i\right) \nu_{k}(t-L) \\
& +P\left(\sum_{j=0}^{L} D_{t-j}-\hat{R}_{t}=S-i\right) \sum_{k=-\infty}^{S-1} \nu_{k}(t-L)
\end{aligned}
$$

- $\mathrm{t}=\mathrm{T}$

For $t=T$ the random variable $\hat{R}_{t}$ in (37) is equal to 0 and we get

$$
\begin{aligned}
P\left(X_{T}=i\right)= & \sum_{k=S}^{\infty} P\left(\sum_{j=0}^{L} D_{T-j}=k-i\right) \nu_{k}(T-L) \\
& +P\left(\sum_{j=0}^{L} D_{T-j}=S-i\right) \sum_{k=-\infty}^{S-1} \nu_{k}(T-L)
\end{aligned}
$$




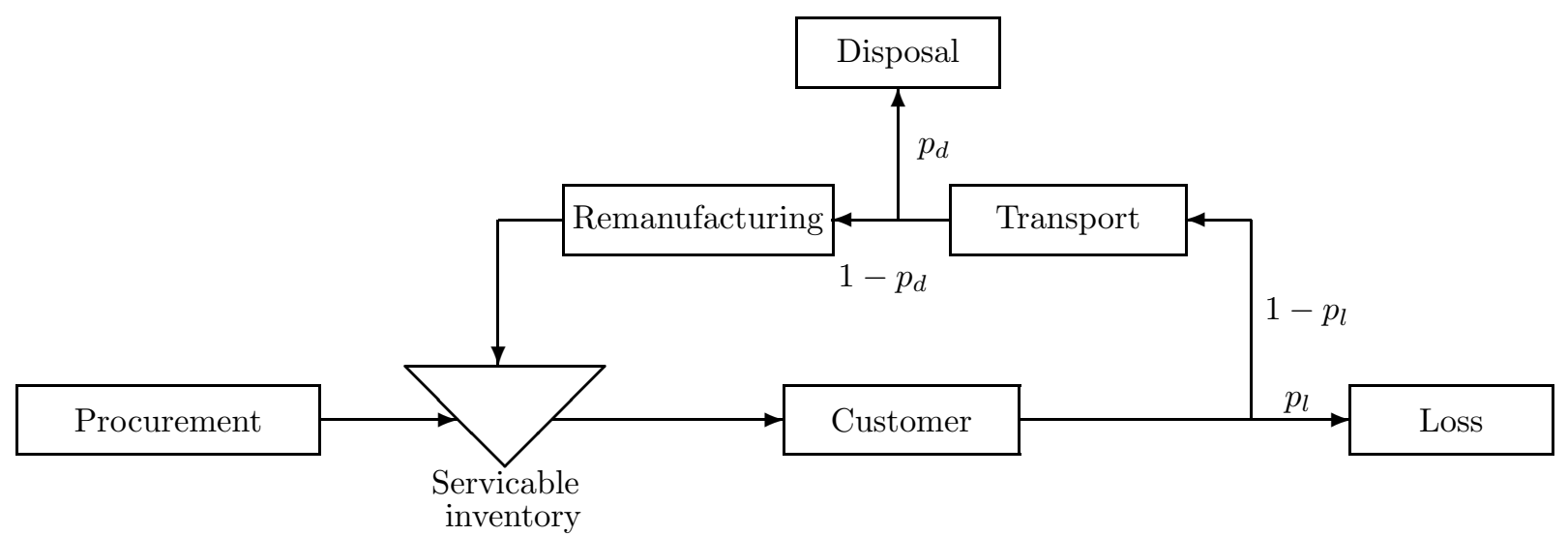

Figure 1: The reuse network

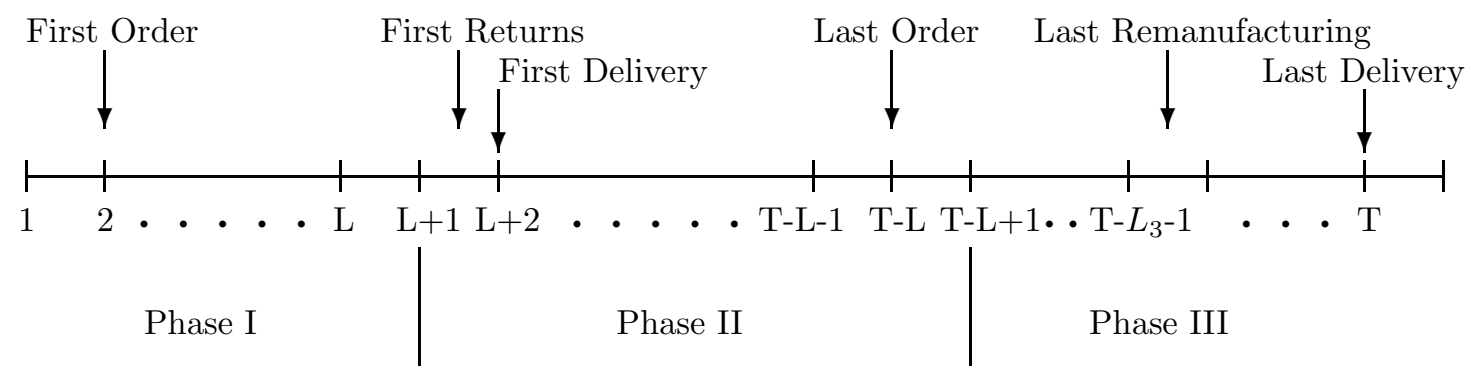

Figure 2: The time phases 

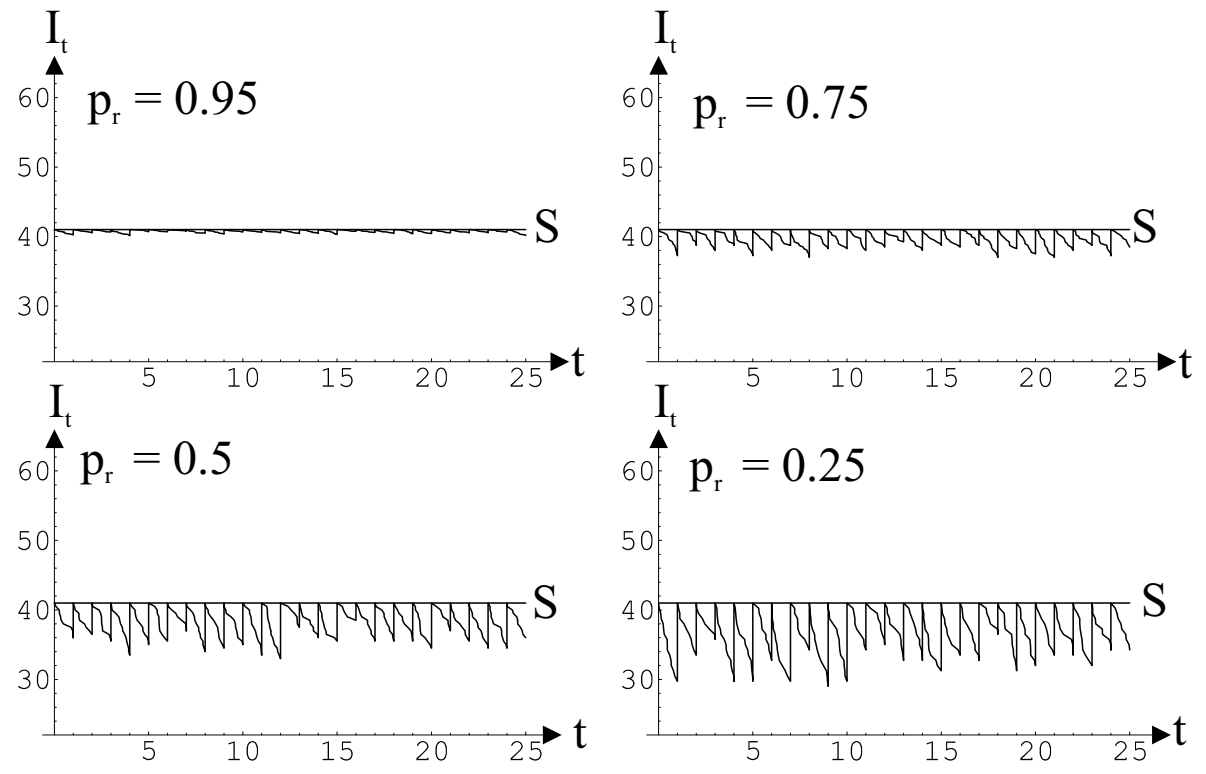

Figure 3: Inventory position for dependent returns; $\lambda_{t}=10, S=A=41$ 

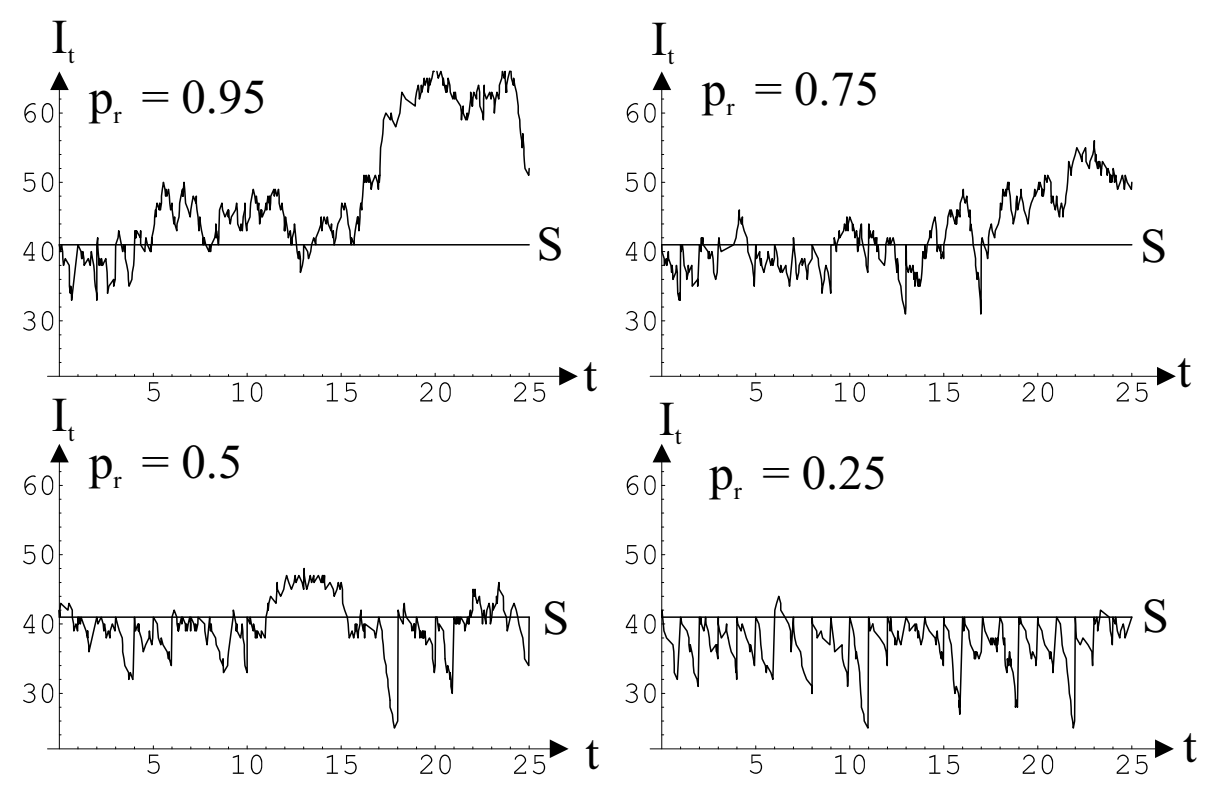

Figure 4: Inventory position for independent returns; $\lambda_{t}=10, S=A=41$
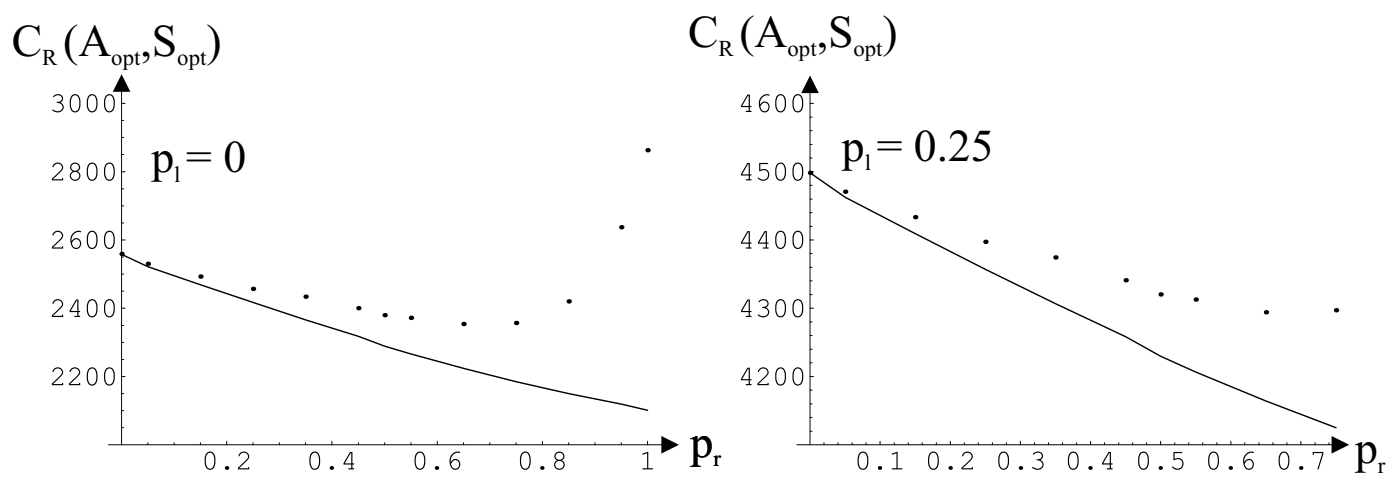

Figure 5: Minimal average relevant costs for the dependent case (solid line) and the independent case (dotted line) as a function of $p_{r}$ 


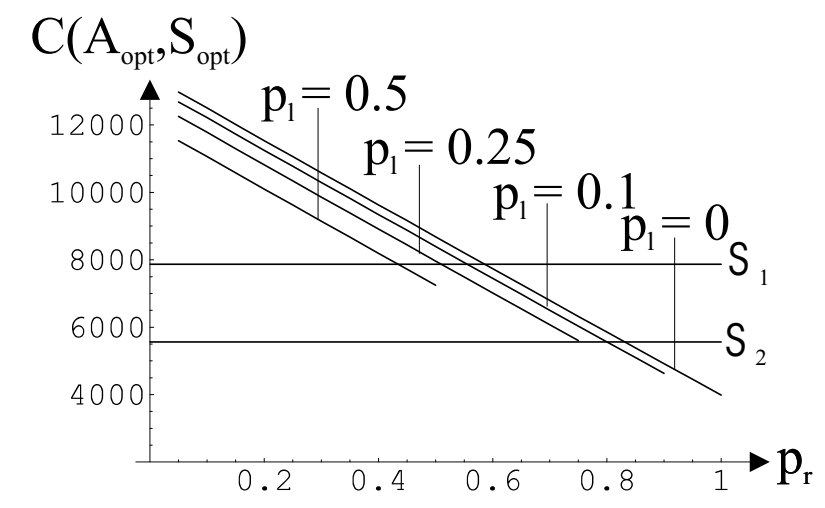

Figure 6: Minimal average costs as a function of $p_{r} ; c_{T}=5, c_{D}=7$
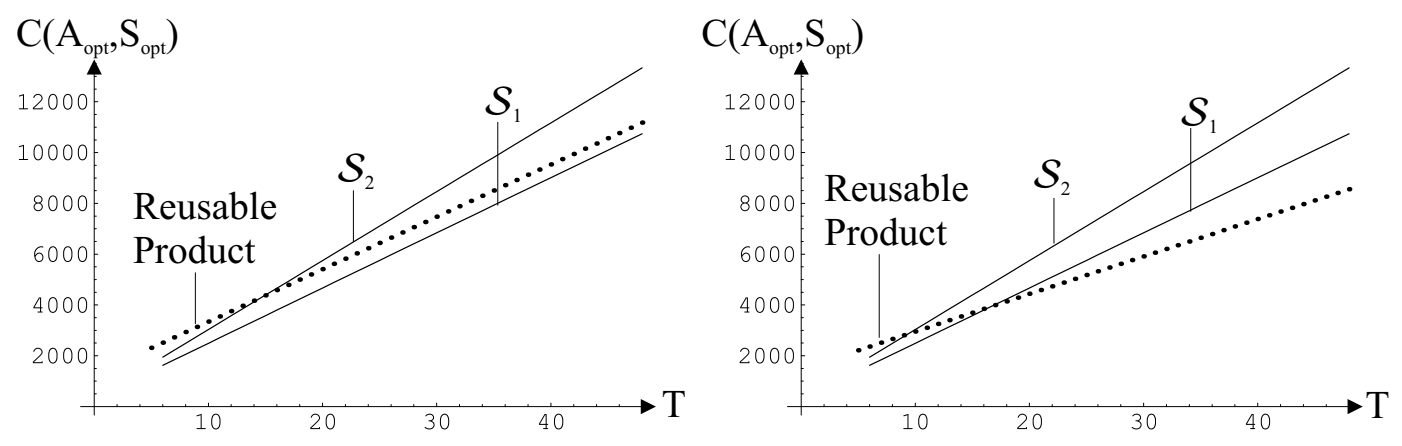

Figure 7: Minimal average costs as a function of $T$ for $p_{r}=0.6$ (left) and $p_{r}=0.75$ (right) 


\begin{tabular}{|c|c|c|}
\hline & dependent & independent \\
\hline$p$ & $V A R\left[D_{t}-\hat{R}_{t}\right]$ & $V A R\left[D_{t}-\hat{R}_{t}\right]$ \\
\hline 0.95 & 0.5 & 19.5 \\
0.75 & 2.5 & 17.5 \\
0.50 & 5.0 & 15.0 \\
0.25 & 7.5 & 12.5 \\
\hline
\end{tabular}

Table 1: Variance of $D_{t}-\hat{R}_{t}$

\begin{tabular}{|c|c|c|c|c|c|c|c|}
\hline & \multicolumn{5}{|c|}{$p_{l}=0$} \\
\hline & \multicolumn{3}{|c|}{ Dependent } & \multicolumn{3}{c|}{ Independent } & Relative difference \\
\hline$p_{r}$ & $A_{\text {opt }}$ & $S_{\text {opt }}$ & $C_{R}\left(A_{\text {opt }}, S_{\text {opt }}\right)$ & $A_{\text {opt }}$ & $S_{\text {opt }}$ & $C_{R}\left(A_{o p t}, S_{\text {opt }}\right)$ & \\
\hline 1.00 & 40 & 40 & 2102 & 42 & 42 & 2863 & $36.2 \%$ \\
0.75 & 42 & 42 & 2185 & 44 & 44 & 2357 & $7.9 \%$ \\
0.50 & 45 & 45 & 2290 & 46 & 46 & 2380 & $3.9 \%$ \\
0.25 & 48 & 48 & 2417 & 48 & 48 & 2457 & $1.6 \%$ \\
0.00 & 51 & 51 & 2558 & 51 & 51 & 2559 & $0.0 \%$ \\
\hline
\end{tabular}

Table 2: Optimal policy and minimal average relevant costs

\begin{tabular}{|c|c|c|c|c|c|c|c|}
\hline & \multicolumn{7}{|c|}{$p_{l}=0.25$} \\
\hline & \multicolumn{3}{|c|}{ Dependent } & \multicolumn{3}{|c|}{ Independent } & Relative difference \\
\hline$p_{r}$ & $A_{o p t}$ & $S_{o p t}$ & $C_{R}\left(A_{o p t}, S_{o p t}\right)$ & $A_{\text {opt }}$ & $S_{\text {opt }}$ & $C_{R}\left(A_{o p t}, S_{o p t}\right)$ & \\
\hline 0.75 & 42 & 42 & 4125 & 44 & 44 & 4297 & $4.2 \%$ \\
\hline 0.50 & 45 & 45 & 4230 & 46 & 46 & 4320 & $2.1 \%$ \\
\hline 0.25 & 48 & 48 & 4357 & 48 & 48 & 4397 & $0.9 \%$ \\
\hline 0.00 & 51 & 51 & 4498 & 51 & 51 & 4498 & $0.0 \%$ \\
\hline
\end{tabular}

Table 3: Optimal policy and minimal average relevant costs 


\begin{tabular}{|c|c|c|c|c|c|c|c|}
\hline & \multicolumn{6}{|c|}{$p_{r}=0.95$} \\
\hline & \multicolumn{3}{|c|}{ Dependent } & \multicolumn{3}{|c|}{ Independent } & Relative difference \\
\hline$T$ & $A_{\text {opt }}$ & $S_{\text {opt }}$ & $C_{R}\left(A_{\text {opt }}, S_{\text {opt }}\right)$ & $A_{\text {opt }}$ & $S_{\text {opt }}$ & $C_{R}\left(A_{\text {opt }}, S_{\text {opt }}\right)$ & \\
\hline 10 & 40 & 40 & 1900 & 42 & 42 & 2157 & $13.5 \%$ \\
20 & 40 & 40 & 2056 & 42 & 42 & 2509 & $22.0 \%$ \\
30 & 40 & 41 & 2208 & 42 & 42 & 2825 & $27.9 \%$ \\
40 & 40 & 41 & 2354 & 42 & 42 & 3129 & $32.9 \%$ \\
48 & 40 & 41 & 2471 & 42 & 42 & 3368 & $36.3 \%$ \\
\hline
\end{tabular}

Table 4: Optimal policy and minimal average relevant costs

\begin{tabular}{|c|c|c|c|c|c|c|c|c|c|}
\hline & \multicolumn{3}{|c|}{ Analytical model } & \multicolumn{3}{|c|}{ Simulation without estimation } & \multicolumn{3}{|c|}{ Simulation with estimation } \\
\hline$p_{r}$ & $A_{\text {opt }}$ & $S_{\text {opt }}$ & $C_{R}\left(A_{\text {opt }}, S_{\text {opt }}\right)$ & $A_{\text {opt }}$ & $S_{\text {opt }}$ & $C_{R}\left(A_{\text {opt }}, S_{\text {opt }}\right)$ & $A_{\text {opt }}$ & $S_{\text {opt }}$ & $C_{R}\left(A_{\text {opt }}, S_{\text {opt }}\right)$ \\
\hline 1.00 & 43 & 43 & 2169 & 43 & 45 & 2272 & 44 & 43 & 2238 \\
0.90 & 45 & 45 & 2249 & 48 & 45 & 2286 & 45 & 45 & 2337 \\
0.80 & 46 & 46 & 2290 & 47 & 46 & 2325 & 46 & 46 & 2290 \\
0.70 & 47 & 47 & 2332 & 47 & 47 & 2429 & 47 & 47 & 2356 \\
0.60 & 48 & 48 & 2377 & 49 & 48 & 2428 & 48 & 48 & 2458 \\
0.50 & 49 & 49 & 2423 & 50 & 50 & 2385 & 50 & 50 & 2457 \\
0.40 & 51 & 51 & 2526 & 51 & 51 & 2350 & 52 & 51 & 2594 \\
0.30 & 51 & 52 & 2575 & 54 & 52 & 2543 & 52 & 52 & 2612 \\
0.20 & 53 & 53 & 2625 & 53 & 53 & 2698 & 54 & 53 & 2660 \\
0.10 & 54 & 54 & 2675 & 54 & 54 & 2679 & 56 & 54 & 2662 \\
0.00 & 55 & 55 & 2726 & 55 & 55 & 2796 & 56 & 56 & 2763 \\
\hline
\end{tabular}

Table 5: Optimal policy and minimal average relevant costs; $\lambda=10, p_{l}=0$

\begin{tabular}{|c|c|c|c|c|c|c|c|c|c|}
\hline & \multicolumn{3}{|c|}{ Analytical model } & \multicolumn{3}{|c|}{ Simulation without estimation } & \multicolumn{3}{|c|}{ Simulation with estimation } \\
\hline$p_{r}$ & $A_{\text {opt }}$ & $S_{\text {opt }}$ & $C_{R}\left(A_{\text {opt }}, S_{\text {opt }}\right)$ & $A_{\text {opt }}$ & $S_{\text {opt }}$ & $C_{R}\left(A_{\text {opt }}, S_{\text {opt }}\right)$ & $A_{\text {opt }}$ & $S_{\text {opt }}$ & $C_{R}\left(A_{\text {opt }}, S_{\text {opt }}\right)$ \\
\hline 1.00 & 341 & 341 & 17899 & 341 & 342 & 18309 & 341 & 344 & 18397 \\
0.90 & 352 & 352 & 17840 & 352 & 352 & 18102 & 352 & 357 & 18176 \\
0.80 & 362 & 362 & 17786 & 364 & 362 & 18061 & 362 & 362 & 18015 \\
0.70 & 373 & 373 & 17758 & 374 & 374 & 18143 & 374 & 374 & 18133 \\
0.60 & 384 & 384 & 17787 & 385 & 385 & 18145 & 384 & 386 & 18261 \\
0.50 & 394 & 394 & 17890 & 395 & 394 & 18350 & 394 & 394 & 18455 \\
0.40 & 405 & 405 & 18134 & 406 & 406 & 18597 & 405 & 405 & 18767 \\
0.30 & 415 & 415 & 18458 & 417 & 416 & 18934 & 416 & 415 & 19084 \\
0.20 & 426 & 426 & 18918 & 426 & 427 & 19424 & 428 & 426 & 19470 \\
0.10 & 437 & 437 & 19436 & 437 & 438 & 19867 & 437 & 437 & 19926 \\
0.00 & 447 & 447 & 19922 & 447 & 447 & 20481 & 448 & 447 & 20355 \\
\hline
\end{tabular}

Table 6: Optimal policy and minimal average relevant costs; $\lambda=100, p_{l}=0$ 



\title{
Publications in the Report Series Research* in Management
}

\author{
ERIM Research Program: "Business Processes, Logistics and Information Systems"
}

\section{1}

Bankruptcy Prediction with Rough Sets

Jan C. Bioch \& Viara Popova

ERS-2001-11-LIS

Neural Networks for Target Selection in Direct Marketing

Rob Potharst, Uzay Kaymak \& Wim Pijls

ERS-2001-14-LIS

An Inventory Model with Dependent Product Demands and Returns

Gudrun P. Kiesmüller \& Erwin van der Laan

ERS-2001-16-LIS

2000

A Greedy Heuristic for a Three-Level Multi-Period Single-Sourcing Problem

H. Edwin Romeijn \& Dolores Romero Morales

ERS-2000-04-LIS

Integer Constraints for Train Series Connections

Rob A. Zuidwijk \& Leo G. Kroon

ERS-2000-05-LIS

Competitive Exception Learning Using Fuzzy Frequency Distribution

W-M. van den Bergh \& J. van den Berg

ERS-2000-06-LIS

Models and Algorithms for Integration of Vehicle and Crew Scheduling

Richard Freling, Dennis Huisman \& Albert P.M. Wagelmans

ERS-2000-14-LIS

Managing Knowledge in a Distributed Decision Making Context: The Way Forward for Decision Support Systems Sajda Qureshi \& Vlatka Hlupic

ERS-2000-16-LIS

Adaptiveness in Virtual Teams: Organisational Challenges and Research Direction

Sajda Qureshi \& Doug Vogel

ERS-2000-20-LIS

Assessment of Sustainable Development: a Novel Approach using Fuzzy Set Theory

A.M.G. Cornelissen, J. van den Berg, W.J. Koops, M. Grossman \& H.M.J. Udo

ERS-2000-23-LIS

A complete overview of the ERIM Report Series Research in Management:

http://www.ers.erim.eur.nl

ERIM Research Programs:

LIS Business Processes, Logistics and Information Systems

ORG Organizing for Performance

MKT Decision Making in Marketing Management

F\&A Financial Decision Making and Accounting

STR Strategic Renewal and the Dynamics of Firms, Networks and Industries 
Applying an Integrated Approach to Vehicle and Crew Scheduling in Practice Richard Freling, Dennis Huisman \& Albert P.M. Wagelmans

ERS-2000-31-LIS

An NPV and AC analysis of a stochastic inventory system with joint manufacturing and remanufacturing Erwin van der Laan

ERS-2000-38-LIS

Generalizing Refinement Operators to Learn Prenex Conjunctive Normal Forms Shan-Hwei Nienhuys-Cheng, Wim Van Laer, Jan Ramon \& Luc De Raedt ERS-2000-39-LIS

Classification and Target Group Selection bases upon Frequent Patterns Wim Pijls \& Rob Potharst

ERS-2000-40-LIS

Average Costs versus Net Present Value: a Comparison for Multi-Source Inventory Models Erwin van der Laan \& Ruud Teunter ERS-2000-47-LIS

Fuzzy Modeling of Client Preference in Data-Rich Marketing Environments Magne Setnes \& Uzay Kaymak ERS-2000-49-LIS

Extended Fuzzy Clustering Algorithms

Uzay Kaymak \& Magne Setnes

ERS-2000-51-LIS

Mining frequent itemsets in memory-resident databases

Wim Pijls \& Jan C. Bioch

ERS-2000-53-LIS

Crew Scheduling for Netherlands Railways. "Destination: Curstomer" Leo Kroon \& Matteo Fischetti

ERS-2000-56-LIS 